\title{
Foreword to special issue on particle methods for flow modeling in ocean engineering
}

\author{
Peter K. Stansby ${ }^{1}$ • Qingwei Ma ${ }^{2}$
}

Published online: 20 July 2016

(C) Springer International Publishing Switzerland 2016

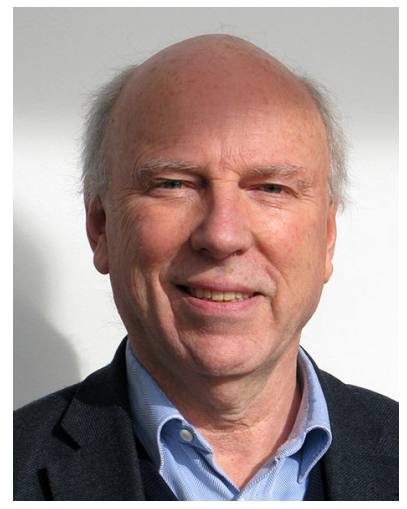

Peter K. Stansby

Ocean engineering poses many problems with violent surface flows and structure interaction. Numerical modelling with particle methods in Lagrangian form has certain advantages over mesh-based methods in that the particles naturally follow the extreme flow distortions which may include multiphase interactions. This special issue has solicited latest developments and applications on particle methods in this context. Smoothed Particle Hydrodynamics (SPH) in its various forms is perhaps most widely applied but there are many variations such as those based on the Meshless Local Petrov-Galerkin (MLPG) method and the Moving Particle Semi-implicit (MPS) method which are also relevant.

Qingwei Ma

q.ma@city.ac.uk

Peter K. Stansby

p.k.stansby@manchester.ac.uk

1 The University of Manchester, Manchester, UK

2 City University London, London, UK

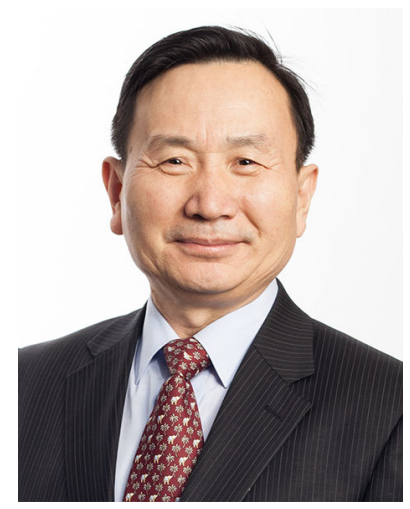

Qingwei Ma

These methods are mainly based on two mathematical formulations: one assumes that the fluid is incompressible and the other assumes that it is weakly compressible, both of which are covered in this special issue. Fundamental aspects of the incompressible formulations for the SPH and MPS methods are reviewed comprehensively in the paper by Gotoh and Khayyer with future perspectives outlined. A key component of all incompressible formulations is the solution of the Poisson's equation for pressure and approaches are reviewed in the paper by Ma et al. for ISPH, MPS and the recent MLPG_R method based on a Rankine source solution.

Incompressible SPH (ISPH) is applied to an oscillating wave surge converter with an OpenMP-based parallel computing approach by Yeylaghi et al. An implicit form of ISPH is applied to solitary wave impact on coastal structures by Sampath et al. A reduced form of ISPH is applied to extreme waves on fixed and moored structures by using FroudeKrylov forcing with analytical added mass by Lind et al., showing accurate results in many cases for little computational expense. The capability of a multi-resolution MPS method for 3D violent free surface flows is demonstrated by Tang et al. 
The most widely used SPH is in weakly compressible form with pressure determined by an equation of state rather than a Poisson's solution. Codes running on GPUs have been applied to tsunami impact on bridge decks by Wei and Dalrymple, and to moored floating bodies by Barreiro et al. They show the versatility of this approach and compatibility with modern computing.

This special issue thus surveys fundamental aspects of particle methods for violent flow and wave-structure interaction modelling, giving latest developments and future trends.
Important applications in ocean (and coastal) engineering are provided showing that the state-of-the-art and novel approaches can already provide realistic predictions.

Peter K. Stansby Qingwei Ma

Guest Editors 